\title{
CRINKLED FUNCTIONS AND INTERSECTIONS WITH POLYNOMIALS
}

\author{
A. M. FINK \\ (Communicated by Andrew M. Bruckner)
}

\begin{abstract}
We prove that if $\left\|f^{(n+1)}\right\|$ is large and $\left\|f^{(n)}\right\|_{\infty}$ is bounded, then there is a polynomial $p$ of degree $n$ such that $f(t)=p(t)$ has many solutions.
\end{abstract}

A classical theorem of Banach (Natanson [1]) says that if $n_{f}(\alpha)=$ the number of sign changes of $\{f(t)-\alpha\}$ on $[0,1]$ and $f$ is a continuous function of bounded variation, then $n_{f}$ is measurable and

$$
\int_{-\infty}^{\infty} n_{f}(\alpha) d \alpha=T V(f)
$$

Assume now that $|f(t)| \leq M$ on $[0,1]$ and $f$ is differentiable. Let $N(f)=$ ess $\sup _{\alpha \in R} n_{f}(\alpha)$. Since $n_{f}(\alpha)=0$ for $|\alpha|>M$ we obtain from (1)

$$
\int_{0}^{1}\left|f^{\prime}(t)\right| d t=\int_{-M}^{M} n_{f}(\alpha) d \alpha \leq 2 M N(f) .
$$

We may state this as an intersection theorem.

Theorem A. Let $f \in C^{1}[0,1]$ and $k$ be a positive integer. If $|f(t)| \leq M$ and $\left\|f^{\prime}\right\|_{1}>2(k-1) M$ then

$$
N(f) \geq k
$$

and there is an $\alpha \in R$ so that the equation $f(t)=\alpha$ has at least $k$ distinct solutions on $[0,1]$. The constant $2(k-1) M$ cannot be replaced by any smaller number and have (3) hold.

Proof. Combining the hypothesis with (2) yields $N(f)>k-1$. Since $N(f)$ is an integer, (3) holds. A piecewise linear function with $f(0)=-M$ and alternate maximum values $M$ and minimum values $-M$, shows that the constant cannot be improved.

This simple result quantifies the intuitive notion that a bounded crinkled function graph must agree with some horizontal line often.

Received by the editors May 20, 1991 and, in revised form, October 25, 1991.

1991 Mathematics Subject Classification. Primary 26D10; Secondary 26A15.

Key words and phrases. Inequalities. 
Schrader in his researches on the compactness problem for $n$th order differential equations asked if there was some version of Theorem A whose hypothesis involved $f^{(n+1)}$ and whose conclusion was about intersection with polynomials of degree $n$. It is our purpose to supply such a theorem.

In some sense, we are looking for a converse of Rolle's theorem. For example, if $f$ agrees with $p(x)=\alpha x+\beta$, a polynomial of degree 1 often, then by Rolle's theorem $f^{\prime}-\alpha$ has many zeroes. By applying Theorem A to $f^{\prime}$ we would have this conclusion if $\left|f^{\prime}\right| \leq M$ and $\left\|f^{\prime \prime}\right\|$ is large. But conversely having $\left\|f^{\prime \prime}\right\|$ large and $\left|f^{\prime}\right| \leq M$ only gets $f^{\prime}-\alpha$ with lots of zeros. Integration does not give $f(x)=\alpha x+\beta$ often. Nevertheless we are able to prove an exact replica of Theorem A in the general case.

Theorem B. For every nonnegative integer $n$ there is a constant $C_{n}>0$ so that for $f \in A C^{(n)}(0,1)$, the inequality

$$
\left\|f^{(n+1)}\right\|_{1}>C_{n} \cdot(k-1) \cdot\left\|f^{(n)}\right\|_{\infty}
$$

implies the existence of a polynomial $p$ of degree $\leq n$ so that the equation $f(t)=p(t)$ has at least $k$ distinct solutions on $[0,1]$.

Let $f \in A C^{(n)}(0,1)$, and put

$$
F_{n}(t, u)=\frac{1}{n !} \int_{u}^{t}(t-s)^{n} f^{(n+1)}(s) d s=f(t)-\sum_{i=0}^{n} \frac{f^{(i)}(u)}{i !}(t-u)^{i}
$$

for every $u, t \in[0,1]$. Then for any fixed $u$, the equation $f_{n}(t, u)=\alpha$ is the equation $f(t)=p(t)$ where $p$ is a polynomial of degree $n$ in the variable $t$. If $n_{(f, n)}(\alpha)$ denotes the number of sign changes in $F_{n}(t, u)=\alpha$, then Theorem A reads

$$
\int_{-\infty}^{\infty} n_{(f, n)}(\alpha) d \alpha=\int_{0}^{1}\left|\frac{d}{d t} F_{n}(t, u)\right| d t=\frac{1}{(n-1) !} \int_{0}^{1}\left|\int_{u}^{t}(t-s)^{n-1} f^{(n+1)}(s) d s\right| d t .
$$

We choose to integrate with respect to $u$ also to get

$$
\int_{0}^{1} \int_{-\infty}^{\infty} n_{(f, n)}(\alpha) d \alpha d u=\frac{1}{(n-1) !} \int_{0}^{1} \int_{1}^{1}\left|\int_{u}^{t}(t-s)^{n-1} f^{(n+1)}(s) d s\right| d t d u .
$$

We want to relate this integral to $\left\|f^{(n+1)}\right\|_{1}$. To do this define

$$
T g=\int_{0}^{1} \int_{0}^{1}\left|\int_{u}^{t}(t-s)^{n-1} g(s) d s\right| d t d u .
$$

Proposition. Let $B=\left\{g \in L^{2}[0,1] \mid\|g\|_{2}=1\right\}$ and $d_{n}=\inf _{g \in B} T g$. Then there is a $g_{0} \in B$ such that $d_{n}=T g_{0}$ and $d_{n}>0$.

Proof. Let $g_{i} \in B$ such that $T g_{i} \rightarrow d_{n}$. By weak compactness of $B$ we may assume that $g_{i}$ converges weakly to $g_{0} \in B$, i.e., $\lim _{i \rightarrow \infty} \int_{0}^{1} g_{i} f d s=\int_{0}^{1} g_{0} f d s$ for all $f \in L^{2}[0,1]$. Now

$$
\int_{u}^{t}(t-s)^{n-1} g_{i}(s) d s=\int_{0}^{1}(t-s)^{n-1} \chi_{[u, t]} g_{i}(s) d s
$$


and so

$$
\int_{u}^{t}(t-s)^{n-1} g_{i}(s) d s \rightarrow \int_{u}^{t}(t-s)^{n-1} g_{0}(s) d s
$$

for every $t, u \in[0,1]$. Since $\left|\int_{u}^{t}(t-s)^{n-1} g_{i}(s) d s\right| \leq\left\|g_{i}\right\|_{1} \leq\left\|g_{i}\right\|_{2}=1$, by the Lebesgue dominated convergence theorem we have $\lim _{i} T g_{i}=T g_{0}=d_{n}$. We now show that $d_{n}>0$.

If $d_{n}=0$ then by (7)

$$
\int_{u}^{t}(t-s)^{n-1} g_{0}(s) d s=0 \quad(t, u \in[0,1]) .
$$

Since $g_{0} \in L^{2} \subset L^{1}$, there is $f \in A C^{(n)}(0,1)$ such that $f^{(n+1)}=g_{0}$ a.e. Then

$$
f(t)-\sum_{i=0}^{n-1} \frac{f^{(i)}(u)}{i !}(t-u)^{i}=\frac{1}{(n-1) !} \int_{u}^{t}(t-s)^{n-1} f^{(n+1)}(s) d s=0
$$

for every $t$ and $u$ and thus $f$ is a polynomial of degree $\leq n$. Therefore $g_{0}=f^{(n+1)}=0$ a.e., contradicting the equation $\left\|g_{0}\right\|_{2}=1$.

Proof of Theorem B. For $n=0$ Theorem B is Theorem A so we may assume $n \geq 1$. Let $C_{n}=4 / d_{n}$ and $\left\|f^{(n+1)}\right\|_{1}>C_{n} \cdot(k-1) \cdot\left\|f^{(n)}\right\|_{\infty}$. Then by the Cauchy-Schwarz inequality

$$
\left\|f^{(n+1)}\right\|_{1} \leq\left\|f^{(n+1)}\right\|_{2} \quad \text { so }\left\|f^{(n+1)}\right\|_{2}>C_{n} \cdot(k-1) \cdot\left\|f^{(n)}\right\|_{\infty} .
$$

Combining (8), (6), and the proposition, we obtain

$$
\int_{0}^{\infty} \int_{-\infty}^{\infty} n_{(f, n)}(\alpha) d \alpha d u \geq \frac{d_{n}}{n !}\left\|f^{(n+1)}\right\|_{2}>\frac{4}{n !}\left\|f^{(n)}\right\|_{\infty} \cdot(k-1) .
$$

Since, by (5),

$$
F_{n}(t, u)=\frac{f^{(n)}(\xi)}{n !}(t-u)^{n}-\frac{f^{(n)}(u)}{n !}(t-u)^{n}
$$

we have $\left|F_{n}(t, u)\right| \leq \frac{2}{n !}\left\|f^{(n)}\right\|_{\infty}$. The left-hand side of (9) may be estimated by $\frac{4}{n !}\left\|f^{(n)}\right\|_{\infty} \cdot \operatorname{ess} \sup n_{(f, n)}(\alpha)$. Thus ess $\sup n_{(f, n)}>k-1$, and the result follows.

Results complementary to ours are given in Agronsky et al. [2] who show that if intersections with polynomials are few, then certain derivatives must be monotone on subintervals.

The author acknowledges that this paper was improved by suggestions of the referee. In the first version, (5) was replaced by a divided difference. The present form makes the argument clearer.

\section{REFERENCES}

1. I. P. Natanson, Konstructive funktionentheorie, Berlin, 1955.

2. S. Agronsky, A. M. Bruckner, M. Laczkovich, and D. Preiss, Convexity conditions and intersections with smooth functions, Trans. Amer. Math. Soc. 289 (1985), 659-677. 